\title{
Thalidomide, dexamethasone and lovastatin with autologous stem cell transplantation as a salvage immunomodulatory therapy in patients with relapsed and refractory multiple myeloma
}

\author{
Marek Hus • Norbert Grzasko • Marta Szostek • Andrzej Pluta • Grzegorz Helbig • \\ Dariusz Woszczyk • Maria Adamczyk-Cioch • Dariusz Jawniak • Wojciech Legiec • \\ Marta Morawska • Justyna Kozinska • Piotr Waciński • Anna Dmoszynska
}

Received: 30 September 2010 / Accepted: 7 June 2011 / Published online: 23 June 2011

(C) The Author(s) 2011. This article is published with open access at Springerlink.com

\begin{abstract}
The treatment of patients with multiple myeloma usually includes many drugs including thalidomide, lenalidomide and bortezomib. Lovastatin and other inhibitors of HMG-CoA reductase demonstrated to exhibit antineoplasmatic and proapoptotic properties in numerous in vitro studies involving myeloma cell lines. We treated 91 patients with relapsed or refractory multiple myeloma with thalidomide, dexamethasone and lovastatin (TDL group, 49 patients) or thalidomide and dexamethasone (TD group,
\end{abstract}

M. Hus $(\bowtie) \cdot$ N. Grzasko $\cdot$ M. Adamczyk-Cioch • D. Jawniak •

W. Legiec $\cdot$ M. Morawska $\cdot$ J. Kozinska $\cdot$ A. Dmoszynska

Department of Haematology and Bone Marrow Transplantation,

Medical University of Lublin,

Staszica 11,

20-081 Lublin, Poland

e-mail: markhus@o2.pl

M. Szostek

Department of Haematology, Collegium Medicum,

Jagiellonian University of Cracow,

Cracow, Poland

A. Pluta

Department of Haematology,

Podkarpackie Oncology Center Hospital,

Brzozow, Poland

G. Helbig

Department of Haematology, Silesian Medical University,

Katowice, Poland

D. Woszczyk

Department of Haematology, State Hospital,

Opole, Poland

P. Waciński

Department of Cardiology, Medical University of Lublin,

Lublin, Poland
42 patients). A clinical response defined of at least $50 \%$ reduction of monoclonal band has been observed in $32 \%$ of TD patients and $44 \%$ of TDL patients. Prolongation of overall survival and progression-free survival in the TDL group as compared with the TD group has been documented. The TDL regimen was safe and well tolerated. The incidence of side effects was comparable in both groups. Plasma cells have been cultured in vitro with thalidomide and lovastatin to assess the impact of both drugs on the apoptosis rate of plasma cells. In vitro experiments revealed that the combination of thalidomide and lovastatin induced higher apoptosis rate than apoptosis induced by each drug alone. Our results suggest that the addition of lovastatin to the TD regimen may improve the response rate in patients with relapsed or refractory myeloma.

Keywords Thalidomide $\cdot$ Lovastatin $\cdot$ Multiple myeloma Apoptosis

\section{Introduction}

Multiple myeloma (MM) is a malignant disorder characterised by an uncontrolled clonal proliferation of malignant plasma cells which produce a monoclonal paraprotein. MM is the second most common haematological malignancy with an annual incidence of approximately 30,000 patients in Europe, resulting in 16,000 deaths yearly. Several combination chemotherapy regimens have been implemented, but still median survival was not significantly affected and remains short-3-4 years for older patients and approaching 6-6.5 years for younger patients eligible for high-dose therapy and autologous transplantation [1-3]. 
MM remains an incurable disease despite the implementation of new agents: thalidomide (THAL), lenalidomide and bortezomib [4, 5]. Monotherapy with thalidomide approaches a $25 \%$ response rate in refractory and de novo treated myeloma patients. Dexamethasone (DEX) inclusive multidrug regimens result in $40-55 \%$ response rate of patients in relapse or resistant to either agent [6]. Lovastatin (LOV) and other inhibitors of HMG-CoA reductase (the rate-limiting enzyme of the mevalonate pathway) have been demonstrated to exhibit antineoplasmatic and proapoptotic properties in numerous in vitro studies involving myeloma cell lines [7-9]. The downstream molecular mechanism of statins inducing apoptosis in tumour cells involves the depletion of geranylgerany, an end product of the mevalonate pathway and a substrate used in protein isoprenylation $[10,11]$. LOV and other statins effectively suppressed the production of vascular endothelial growth factor by tumour cells harbouring RAS mutations in vitro [12].

This observation stimulated us to administer LOV in combination with THAL and DEX in refractory multiple myeloma patients. The results of our experience with THAL and LOV therapy in patients with relapsed or refractory MM have been reported.

\section{Design and methods}

Ninety-one patients with relapsed or refractory multiple myeloma were enrolled at four Polish haematology centres and randomly assigned to receive THAL, DEX and LOV (TDL group) or THAL and DEX (TD group). Patients' characteristics prior to the start of treatment are summarised in Table 1. We compared TDL and TD groups in terms of: age, disease stage according to the International Staging System (ISS), renal function, blood morphology, bone marrow plasma cells number, as well as serum total protein, monoclonal band, albumins, beta-2-microglobulin, Creactive protein and LDH level. Chromosomal studies have not been performed in the majority of patients; therefore, these results will not be analysed. We did not observe statistically significant differences between compared parameters in analysed patient groups except serum urea level, which was lower in TDL than in the TD group (median $38.7 \mathrm{mg} /$ day versus $48.3 \mathrm{mg} / \mathrm{dL}, p=0.04$ ), although serum creatinine concentrations were not significantly different.

Treatment groups consisted of adult ( $>18$ years) patients with relapsed or refractory myeloma. Measurable disease has been defined as a serum monoclonal immunoglobulin concentration of at least $10 \mathrm{~g} / \mathrm{L}$ for IgG or $5 \mathrm{~g} / \mathrm{L}$ for IgA in patients with relapsed or refractory myeloma, or urinary light chains excretion of at least $200 \mathrm{mg}$ per $24 \mathrm{~h}$. Patients with nonsecretory $\mathrm{MM}$ had respective measurable disease
Table 1 Patients' characteristics

\begin{tabular}{|c|c|c|}
\hline & $\operatorname{TLD}(n=49)$ & $\mathrm{TD}(n=42)$ \\
\hline Age & $59.3 \pm 7.7$ & $62.4 \pm 8.9$ \\
\hline \multirow[t]{2}{*}{ Sex } & Female, 25 & Female, 22 \\
\hline & Male, 24 & Male, 20 \\
\hline Patients resistant to chemotherapy & 5 & 4 \\
\hline Relapsed patients & 44 & 38 \\
\hline Chemotherapy lines/courses (median) & $2 / 8$ & $2 / 9$ \\
\hline \multirow[t]{5}{*}{ Type of myeloma } & $\operatorname{IgG} 28$ & $\operatorname{IgG} 24$ \\
\hline & $\operatorname{IgA}, 15$ & $\operatorname{Ig} \mathrm{A}, 12$ \\
\hline & $\begin{array}{r}\text { Light chain } \\
\text { disease, } 2\end{array}$ & $\begin{array}{r}\text { Light chain } \\
\text { disease, } 4\end{array}$ \\
\hline & $\begin{array}{l}\text { Nonsecretory, } \\
2\end{array}$ & $\begin{array}{l}\text { Nonsecretory, } \\
1\end{array}$ \\
\hline & Solitare, 2 & Solitare, 1 \\
\hline \multirow[t]{2}{*}{ Light chain } & Kappa, 38 & Kappa, 32 \\
\hline & Lambda, 11 & Lambda, 10 \\
\hline \multicolumn{3}{|l|}{ Cytogenetics } \\
\hline Isolated del(17p13) & 4 & 3 \\
\hline $\begin{array}{l}\text { Complex abnormalities: } \mathrm{t}(4 ; 14) \text {, del } \\
\text { (17p13), del (13q14) }\end{array}$ & 3 & 3 \\
\hline Without abnormalities & 7 & 6 \\
\hline No data & 35 & 30 \\
\hline Marrow plasma cells (\%) & $43.7 \pm 27.4$ & $49.2 \pm 26.3$ \\
\hline $\mathrm{CRP}(\mathrm{mg} / \mathrm{L})$ & $11.4 \pm 30.5$ & $9.4 \pm 14.2$ \\
\hline B2M (mg/L) & $3.7 \pm 3.6$ & $3.58 \pm 3.7$ \\
\hline LDH (IU/L) & $296.4 \pm 160.9$ & $282.6 \pm 142.1$ \\
\hline Creatinine (mg/dL) & $1.0 \pm 0.5$ & $1.6 \pm 1.8$ \\
\hline \multicolumn{3}{|l|}{ ISS prognostic index } \\
\hline ISS 1 & 5 & 4 \\
\hline ISS 2 & 38 & 31 \\
\hline ISS 3 & 6 & 7 \\
\hline
\end{tabular}

activity assessment (immunophenotyping, MRI). Beta-2 microglobulin and albumin studies have been performed in all patients. The ISS protocol investigation staging system has been implemented. All patients had relapsed following previous one to two lines of chemotherapy or were resistant to treatment with at least one to two lines of chemotherapy. Eligibility criteria included WHO performance status of $0-2$, serum ASPAT or ALAT no higher than two times the upper limit of normal, serum creatinine level less than $2 \mathrm{mg} / \mathrm{dL}$, platelet count of at least $50 \mathrm{G} / \mathrm{L}$ and absolute neutrophil count of at least to $1.0 \mathrm{G} / \mathrm{L}$. Exclusion criteria included clinically relevant infection (e.g. hepatitis $\mathrm{B}$ or $\mathrm{C}$ ), serious comorbidities or prior malignancy. Patients consented to use mechanical contraception during the study period. Only patients with a negative urine pregnancy test have been included in the study. All patients gave written informed consent before entering the study. The study has been performed according to the Declaration of Helsinki, 
under the auspices of protocol approved by the institutional ethics committee.

\section{Therapeutic protocol}

Patients received drugs orally in 28-day cycles. THAL was given from day 1 to day 28 of each cycle at a dose of $100 \mathrm{mg}$ at bedtime. DEX was administered at a dose of $20 \mathrm{mg}$ daily on days 1-4 of each cycle. LOV was administered at a dose of $2 \mathrm{mg} / \mathrm{kg}$ on days $1-5$ and $8-12$ and at a dose of $0.5 \mathrm{mg} / \mathrm{kg}$ on days $15-28$ of each cycle. TDL regimen was administered to 49 patients and the TD regimen to 42 patients. Treatment was discontinued in patients who progressed to TD or TDL and was withheld for grade 3 or worse non-haematological toxicity, or grade 4 haematological toxicity. Aspirin $(75 \mathrm{mg} /$ day) was given as thromboprophylaxis. All patients received $90 \mathrm{mg}$ intravenous pamidronate monthly. The high dose of cyclophosphamide $\left(4 \mathrm{~g} / \mathrm{m}^{2}\right)$ with filgrastim was applied as mobilisation. The conditioning regimen consisted of a high dose of melphalan-200 mg/m².

\section{Assessment of response}

The response to therapy was assessed after 6 cycles according to the modified criteria of the European Group for Blood and Marrow Transplantation. Briefly, a complete response (CR) was defined by the absence of monoclonal protein (M-protein) in the serum and urine confirmed by immunofixation and normal serum free light chain ratio, which was in turn confirmed by repeated measurements after 6 weeks. Near-complete response (NCR) was defined as $\mathrm{CR}$ with positive immunofixation test. A partial response was defined as the reduction of monoclonal protein in serum by at least $50 \%$ and reduction in urine by at least $90 \%$. Progressive disease was defined by an increase of Mprotein in the serum or urine of more than $25 \%$, an increase in bone marrow plasma cells and new hypercalcemia. The toxicity of TDL and TD regimens was assessed according to CTC.

In vitro short-term culture of plasma cells

Mononuclear cells of the patients' bone marrow were isolated by Ficoll-Hypaque density gradient centrifugation. The percentage of plasma cells was evaluated by triple staining (CD138/FITC, CD38/PE and CD45/PC-5) and analysed by flow cytometry. Isolated cells were cultured in Roswell Park Memorial Institute 1640 medium and supplemented with $10 \%$ foetal bovine serum and $10 \mu \mathrm{g} /$ $\mathrm{mL}$ of THAL or $10 \mu \mathrm{M}$ of LOV, or the combination of both drugs in a humidified atmosphere of $5 \% \mathrm{CO}_{2}$ and $37^{\circ} \mathrm{C}$. $\mathrm{LOV}$ in the inactive form was obtained from Merck
Laboratories, USA. It was converted to the active form by dissolving in ethanol, heating at $50^{\circ} \mathrm{C}$ in $0.1 \mathrm{M} \mathrm{NaOH}$ and neutralised with $\mathrm{HCl}$. Distilled water was added to a final concentration of $8 \mathrm{mg} / \mathrm{mL}$. This stock solution was frozen at $-20^{\circ} \mathrm{C}$ and its $1-\mu \mathrm{l}$ aliquots were added per millilitre of cell culture. After 24 and $48 \mathrm{~h}$ of incubation, the cells were washed and stained with annexin V/FITC and propidium iodide to detect apoptosis. The percentage of apoptotic cells was determined by flow cytometry analysis on FACSCalibur $^{\mathrm{TM}}$ using CellQuest ${ }^{\mathrm{TM}}$ software (Fig. 1).

\section{Statistical analysis}

The survival analysis was performed in patients from TDL and TD groups who did not receive high-dose therapy with autologous stem cell transplant (HDT/ASCT) following TDL or TD regimen (31 TDL and 38 TD patients). This allowed us to exclude the HDT/ASCT influence on survival, as the proportion of patients given HDT/ASCT in both groups was not equal. Overall survival (OS) was defined as the time from study entry to death or the last date on which the patient was alive. Progression-free survival (PFS) was defined as the time from study entry to any event such as disease progression or death from any cause. The survival analyses were done by the Kaplan-Meier method. The influence of independent variables on survival was tested by proportional Cox hazard regression. Differences in clinical parameters between the TDL and TD groups were tested by Mann-Whitney $U$ test. Assessment of the impact of drugs in cell culture was performed using Friedman ANOVA test.

The primary objectives of the study were to determine the durations of PFS and OS in both groups of patients. The secondary objectives of this study were to determine the toxicity of thalidomide and dexamethasone in combination with lovastatin and to demonstrate the possibility of stem cell harvesting and autologous bone marrow transplant after treatment with thalidomide, dexamethasone and lovastatin.

\section{Results}

Thirty-two percent of TD and 44\% of TDL patients responded to the treatment. NCR and CR were observed in $5 \%$ and $11 \%$, respectively (Table 2 ). We observed a significant negative correlation between response and bone marrow infiltration $(p<0.005)$. The median time to response was shorter in the TDL group than in the TD group (1.5 versus 3 months, respectively; $p=0.001$ ). Short time to $50 \%$ reduction of M-protein was associated with better response. Among patients who had not been submitted to HDT/ ASCT treatment, patients treated with TDL regimen had median overall survival of 49 versus 39.5 months in TD 
A) Lovastatix

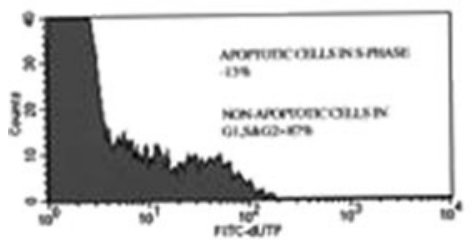

sTMALIDOMDE.
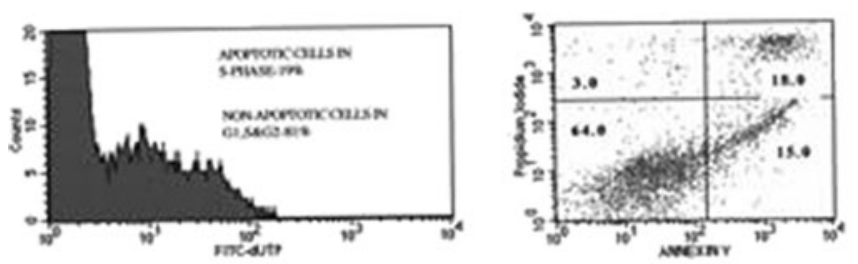

C)LOVASTATIN,THALIDOMIDE
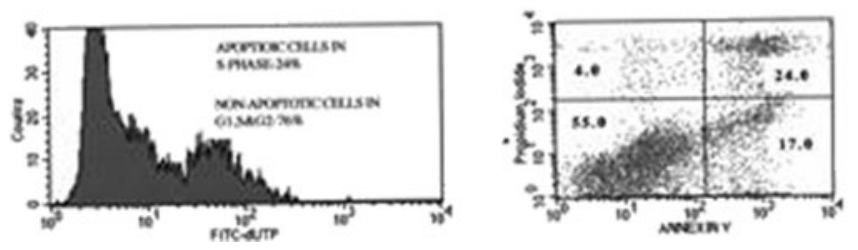

Fig. 1 a-c Thalidomide (THAL) and lovastatin ( $L O V$ ) induced higher apoptosis rate than apoptosis rate induced by each drug alone. The percentage of early apoptotic cells evaluated after 24 and $48 \mathrm{~h}$ of cell culture was higher in the presence of both THAL and LOV. The median percentage of early apoptotic cells after $24 \mathrm{~h}$ of culture was

patients although the difference was not statistically significant. Figure 2a shows the Kaplan-Meier estimate of OS in both groups of patients. The evaluation of PFS in patients without HDT/ASCT showed significant differences. PFS was significantly shorter in patients treated with TD regimen (median 16 months) in comparison to TDL-treated patients (median 33 months, $p=0.04849$ in Wilcoxon-Gehan test). Figure $2 \mathrm{~b}$ presents the KaplanMeier estimate of PFS in both groups of patients.

In 21 (42.8\%) TDL and 7 (16.6\%) TD patients, successful stem cell harvest was performed and the median number of collected CD34+ cells was $8.26 \times 10^{6}$ per $\mathrm{kg}$ in the TDL group and $6.76 \times 10^{6}$ per $\mathrm{kg}$ in the TD group $(p>0.05)$. Successful autologous stem cell transplantation was performed in $18(36.7 \%)$ patients of the TDL group and 4

Table 2 Percentage of responders and non-responders

\begin{tabular}{llcc}
\hline & & $\operatorname{TLD}(n=49)(\%)$ & $\mathrm{TD}(n=42)(\%)$ \\
\hline Clinical response & & 44 & 32 \\
M-protein reduction & $50-75 \%$ & 17 & 17 \\
& $>75 \%$ & 16 & 10 \\
& $>90 \%$ & 11 & 5 \\
No response & $\mathrm{CR}(\mathrm{IF})$ & 7 & 2 \\
\hline
\end{tabular}

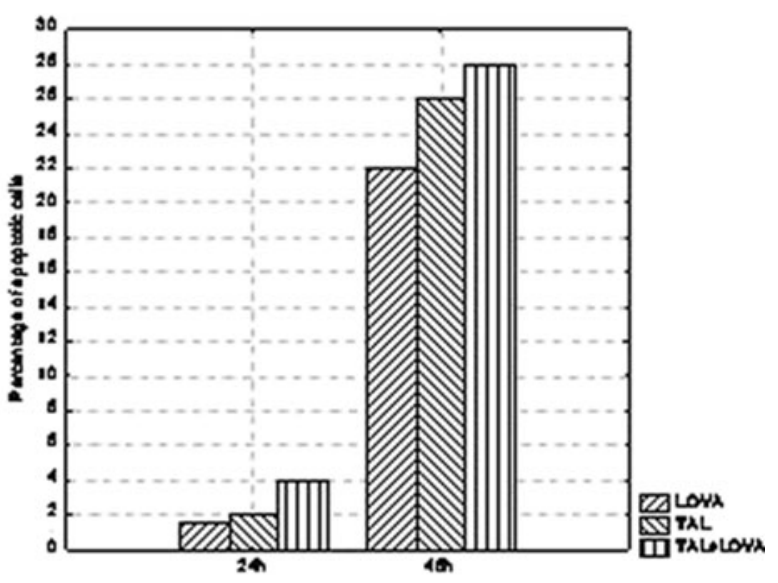

$4 \%$ versus $2 \%$ in the presence of THAL and $1.5 \%$ in the presence of LOV. After $48 \mathrm{~h}$ of culture, the median values were $28 \%, 26 \%$ and $22 \%$, respectively. At both time points, the differences were statistically significant $(p<0.001$ using Friedman ANOVA test)

(9.5\%) of the TD group. The recovery time for WBC $>0.5 \mathrm{~g} /$ 1 and PLT $>20 \mathrm{~g} / 1$ was comparable in the TDL and TD groups ( $p>0.05$ for WBC and PLT). The 100-day transplantrelated mortality was $0 \%$.

\section{Toxicity profile}

The TDL regimen was well tolerated. We did not observe toxic death during the treatment. Common side effects such as somnolence, fatigue and constipation were observed in about $20 \%$ of the patients in both the TDL and TD groups. In four (8.2\%) TDL and two (4.8\%) TD patients, we diagnosed deep vein thrombosis. In one patient in the TDL group, grade 4 pulmonary embolism occurred. We observed grade 3-4 sensory neuropathy in $12(24.3 \%)$ patients in the TDL group and 10 (23.8\%) patients in the TD group. Five (10.2\%) TDL patients were observed with a moderate increase of aminotransferases. No TDL-treated patients were observed with increased myoglobine and troponine following treatment. In three (6.1\%) TDL and two (4.8\%) TD patients, sinus bradycardia was observed. Neutropenia was noted in four $(8.2 \%)$ TDL and four $(9.6 \%)$ TD patients and thrombocytopenia was noted in two $(4.1 \%)$ TDL and two $(4.8 \%)$ TD patients. We did not observe any haematological adverse events in grade 3 or 4 according to CTC. The summary of side effects in grade 3 or 4 is shown in Table 3. 

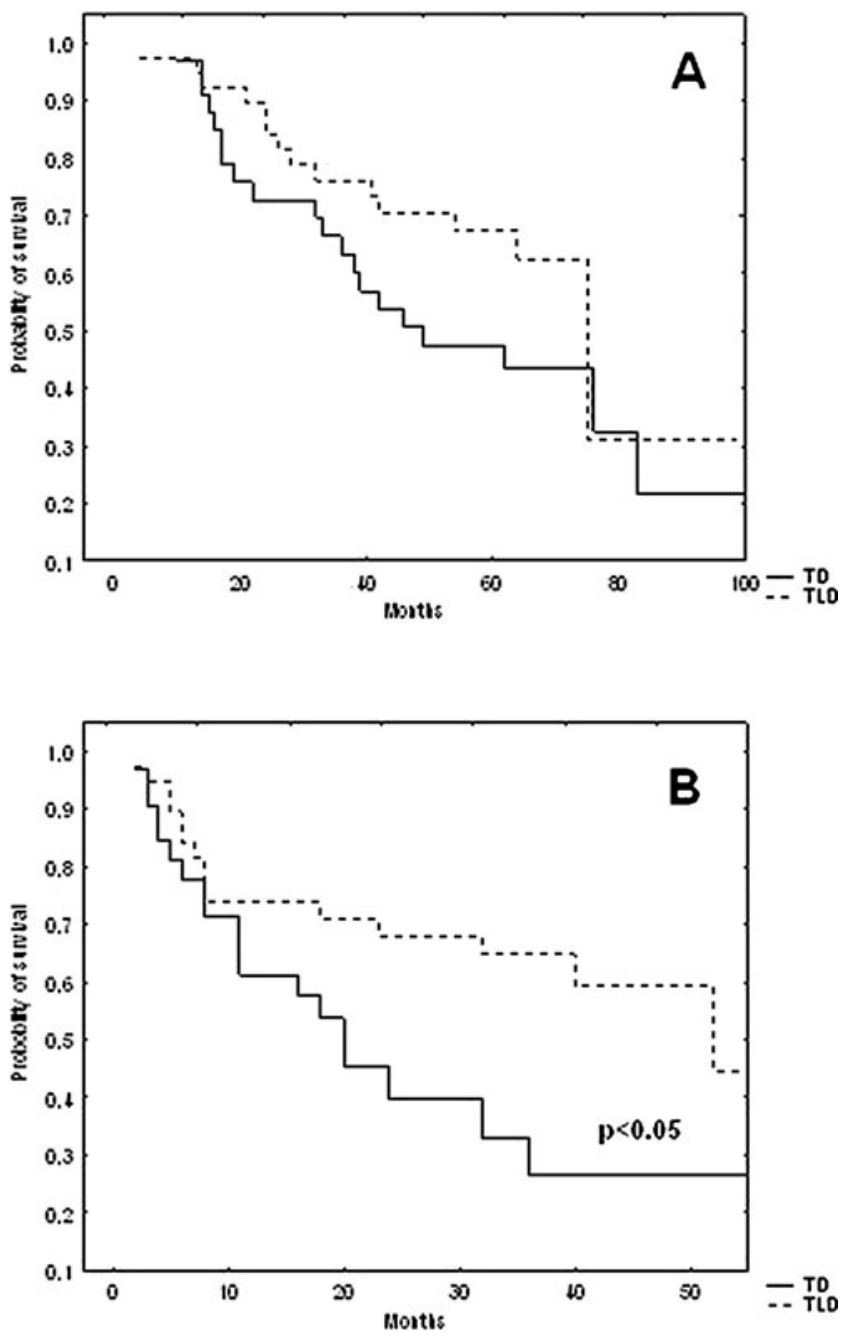

Fig. 2 Overall survival (OS) and progression-free survival (PFS) in both groups of patients including TD or TDL therapy and high-dose melphalan. a Median OS was longer in patients treated with TDL regimen than with TD regimen (47.5 versus 36.5 months, $p=0.073$ ). b Median PFS was significantly longer in the TDL group as compared to the patients treated with TD ( 28.5 versus 6 months, $p=0.0484$ )

\section{Discussion}

This is the first trial comparing TD with TDL efficacy in refractory or relapsed patients with MM. There are only a few publications concerning the activity of LOV in myeloma and its relation to the regulation of apoptosis in MM cells [7, 9, 11]. In our study, the assessment of early apoptotic signals was done in 25 short-term cultures of plasma cells obtained from patients with relapsed MM. Detection of apoptosis after 24 and $48 \mathrm{~h}$ of incubation confirmed that LOV has additional influence on the induction of apoptosis (Fig. 1). Results of in vitro studies of statins against myeloma cells showed that statins' activity in the concentration ranged between 1 and $30 \mu \mathrm{M}$. We assumed, therefore, that only high doses of statins could be beneficial for MM patients. Thibault et al. [13] in a clinical
Table 3 Side effects of treatment

\begin{tabular}{lcc}
\hline CTC & TLD $(n=49)$ & TD $(n=42)$ \\
\hline Neuropathy sensory & $12(24.3 \%)$ & $10(23.8 \%)$ \\
Fatigue (lethargy, malaise or asthenia) & $10(20.4 \%)$ & $8(19.0 \%)$ \\
Constipation & $8(16.3 \%)$ & $6(14.3 \%)$ \\
Somnolence/depressed level & $5(10.2 \%)$ & $4(9.5 \%)$ \\
$\quad$ of consciousness & & \\
Dizziness & $4(8.2 \%)$ & $4(9.5 \%)$ \\
Thrombosis/embolism & $4(8.2 \%)$ & $2(4.8 \%)$ \\
Oedema & $3(6.1 \%)$ & $2(4.8 \%)$ \\
Sick bradycardia & $3(6.1 \%)$ & $2(4.8 \%)$ \\
Allergic reaction/hypersensitivity & $2(4.1 \%)$ & $2(4.8 \%)$ \\
Nausea & $2(4.1 \%)$ & $2(4.8 \%)$ \\
Vomiting & $2(4.1 \%)$ & $1(2.4 \%)$ \\
Fever & $0(0.0 \%)$ & $1(2.4 \%)$ \\
\hline
\end{tabular}

study assessed the tolerability of LOV administered at a dose ranging from 2 to $45 \mathrm{mg} / \mathrm{kg} /$ day with 7-day courses given monthly in patients with various types of cancer. They concluded that the administration of LOV at a dose of $10-25 \mathrm{mg} / \mathrm{kg}$ daily for seven consecutive days was well tolerated by cancer patients. Dmoszynska et al. [14] administered LOV in a patient with relapsed MM at a daily dose of $15 \mathrm{mg} / \mathrm{kg}$ for ten consecutive days monthly for three consecutive months with ubiquinone prophylaxis at a dose of $240 \mathrm{mg}$ daily. They did not report severe adverse events, including cardiomyolysis. Sondergaard et al. [15], in a phase 2 trial, used simvastatin in six patients at a daily dose of $15 \mathrm{mg} / \mathrm{kg}$ in 7-day cycles. The study was prematurely discontinued due to the absence of clinical response and the presence of adverse events.

In our study, 49 patients in the TDL group received LOV at a dose of $1 \mathrm{mg} / \mathrm{kg}$ (55 to $90 \mathrm{mg}$ daily, median $65 \mathrm{mg}$ daily) for 5 days and weekly for two consecutive weeks. Patients received a maintenance dose of $0.5 \mathrm{mg} / \mathrm{kg}$ for the next 2 weeks. We did not observe clinical or biochemical evidence of significant toxicity. Apathy and muscle weakness were observed in about $15 \%$ of the patients both in the TDL and TD groups. It is not clear whether they have been related to dexamethasone or lovastatin therapy. Results in the TDL group showed a significantly higher rate of very good responses (CR and NCR) and significantly shorter time to response in comparison to the TD-treated patients. Comparison between our results and the results reported by different authors using TD regimen is difficult due to different doses of THAL and DEX and different therapy schemes studied. Lilienfeld-Toal et al. [16] conducted a review of 12 studies evaluating TD regimen in refractory and relapsed myeloma. Among 451 patients included in all reviewed studies, the response rate was $46 \%$, six studies reported a median PFS of 8 months (range 3.9 to 12 months) 
and median OS reported by seven studies was 27 months (range 13 to 38 months). In our study, the response rate was lower and reached $32 \%$ of patients achieving at least partial response. Such difference may reflect a higher dose of THAL used in most of the reviewed studies. We used the daily dose of $100 \mathrm{mg}$, whereas such a dose was used only in one study; median starting dose was $200 \mathrm{mg}$ daily and median target dose was $350 \mathrm{mg}$ daily [16]. Murakami et al. [17] treated 66 patients with relatively low dose of THAL and they obtained results comparable to ours. Overall response rate according to the authors' criteria was $63.6 \%$, but only $25.8 \%$ of the patients achieved at least partial response with the reduction of M-protein level by $50 \%$. The median PFS and OS were longer in our patients (16 and 39.5 months, respectively) than in the analysis done by Lilienfeld-Toal et al. [16]. In another study, Palumbo et al. [18] treated 120 patients, administering THAL at a daily dose of $100 \mathrm{mg}$ and DEX at a daily dose of $40 \mathrm{mg}$ for four consecutive days monthly. The results of their studies have similar results to our results. In patients after one line of chemotherapy, the median PFS was 17 months and the median OS was not reached, but the probability of survival of 3 years was $60 \%$. In patients after two or more lines of chemotherapy, the median PFS and OS were 11 and 19 months, respectively. Anagnostopoulos et al. [19] reported the median OS of 38 months. Cibeira et al. [20] reported PFS of 15.6 months and OS of 28.4 months among relapsing MM patients.

In our study, patients treated with TDL regimen had significantly prolonged survival and overall survival (median PFS over 33 months and median OS over 49 months). These results suggest that the addition of LOV to THAL and DEX is safe and improves the response rate in patients with relapsed or refractory $\mathrm{MM}$, without hampering mobilisation efficacy and implementation of high-dose therapy. A future prospective randomised study is needed to confirm the value of LOV or other HMG-CoA reductase inhibitors in the treatment of MM patients.

Open Access This article is distributed under the terms of the Creative Commons Attribution Noncommercial License which permits any noncommercial use, distribution, and reproduction in any medium, provided the original author(s) and source are credited.

\section{References}

1. Blade J, Rosinol L (2008) Advances in therapy of multiple myeloma. Curr Opin Oncol 20:697-704
2. Hus M, Dmoszynska A, Soroka-Wojtaszko M et al (2001) Thalidomide treatment of resistant or relapsed multiple myeloma. Haematologica 86:404-408

3. Singhal S, Mehta J, Desikan R et al (1999) Antitumor activity of thalidomide in refractory multiple myeloma. N Engl J Med 341: $1565-1571$

4. Barlogi B, Shaughnessy J, Tricot G et al (2004) Treatment of multiple myeloma. Blood 103:20-32

5. Palumbo A, Ambrosini MT, Benevolo G et al (2007) Bortezomib, melphalan, prednisone, and thalidomide for relapsed multiple myeloma. Blood 109:2767-2772

6. Corso A, Zappasodi P, Barbarano L et al (2009) Long-term outcome in relapsed and refractory multiple myeloma treated with thalidomide. Balancing efficacy and side-effects. Leukemia Res 23:145e-149e

7. Agarwal B, Halmos B, Feoktistov AS et al (2002) Mechanism of lovastatin-induced apoptosis in intestinal intestinal epithelial cells. Carcinogenesis 23:521-528

8. Wong WW, Clendening JW, Martirosyan A et al (2007) Determinants of sensitivity to lovastatin-induced apoptosis in multiple myeloma. Mol Cancer Ther 6:1886-1897

9. Van de Donk NW, Kamphuis MM, Van Kessel B et al (2003) Inhibition of protein geranylgeranylation induced apoptosis in myeloma plasma cells by reducing Mcl-1 protein levels. Blood 102:3354-3362

10. Baulch-Brown C, Molloy TJ, Yeh SL et al (2007) Inhibitors of the mevalonate pathway as potential therapeutic agents in multiple myeloma. Leuk Res 31:341-352

11. Van de Donk NW, Kamphuls MM, Lokhorst HM et al (2002) The cholesterol lowering drug lovastatin induces cell death in myeloma plasma cells. Leukemia 16:1362-1371

12. Feleszko W, Balkowiec E, Sieberth E et al (1999) Lovastatin and TNF alpha exhibit potentiated antitumor effects against Ha-ras transformer marine tumor via inhibition of tumor-induced angiogenesis. Int J Cancer 81:560-567

13. Thibault A, Samid D, Tompkims AC (1996) Phase I study of lovastatin, an inhibitor of the mevalonate pathway, in patients with cancer. Clin Cancer Res 2:483-491

14. Dmoszynska A, Podhorecka M, Rolinski J et al (2004) Influence of lovastatin on BCL-2 and BAX expression by plasma cells and $\mathrm{T}$ lymphocytes in short-term cultures of multiple myeloma bone marrow mononuclear cells. Pol J Pharmacology 56:485-489

15. Sondergaard TE, Pedersen PT, Andersen TL et al (2009) A phase II clinical trial does not show that high dose simvastatin has beneficial effect on markers of bone turnover in multiple myeloma. Hematol Oncol 27:17-22

16. Lilienfeld-Toal M, Hahn-Ast C, Furkert K et al (2008) A systematic review of phase II trials of thalidomide/dexamethasone combination therapy in patients with relapsed or refractory multiple myeloma. Eur J Haematol 81:247-252

17. Murakami H, Handa H, Abe M et al (2007) Low-dose thalidomide plus low-dose dexamethasone therapy in patients with refractory multiple myeloma. Eur J Haematol 79:234-239

18. Palumbo A, Bertola A, Falco P et al (2004) Efficacy of low-dose thalidomide and dexamethasone as first salvage regimen in multiple myeloma. Hematol J 5:318-334

19. Anagnostopoulos A, Weber D, Rankin K et al (2003) Thalidomide and dexamethasone for resistant multiple myeloma. Br J Haematol 121:768-771

20. Cibeira MT, Rosinol L, Ramiro L et al (2006) Long-term results of thalidomide in refractory and relapsed multiple myeloma with emphasis on response duration. Eur J Haematol 77:486-492 